\title{
Crescimento e acúmulo de fósforo pelo feijoeiro tratado com fosfato e fosfito via foliar
}

\section{Growth and accumulation of phosphorus by bean plants treated with foliar phosphate and phosphite}

\author{
Josinaldo Lopes Araujo ${ }^{1 *}$; Valdemar Faquin²; Fabrício William de Ávila³; \\ Thiago Queiroz Pedroso ${ }^{4}$
}

\begin{abstract}
Resumo
Atualmente o uso de fosfitos na agricultura como fungicida ou como fonte suplementar de fósforo (P) tem sido amplamente difundido; contudo, pouco se sabe sobre seus efeitos na nutrição fosfatada em culturas importantes como o feijoeiro. No presente trabalho objetivou-se avaliar o efeito do fosfito e do fosfato, aplicados via foliar, sobre o crescimento e a nutrição fosfatada do feijoeiro em condições de baixo e adequado suprimento de P. O experimento foi conduzido em delineamento inteiramente casualizado, em esquema fatorial $2 \times 3 \times 2$, combinando-se duas concentrações de fosfato em solução nutritiva ( $1,5 \mathrm{mg} \mathrm{L}^{-1}=$ baixo fósforo e $20 \mathrm{mg} \mathrm{L}^{-1}=$ fósforo adequado), três produtos de aplicação foliar: $\mathrm{KH}_{2} \mathrm{PO}_{3}$ (fosfito monobásico de potássio), $\mathrm{KH}_{2} \mathrm{PO}_{4}$ (fosfato monobásico de potássio) e $\mathrm{KCl}$ (cloreto de potássio) como testemunha e dois tratamentos referentes ao número de aplicações (aplicação única, no surgimento do primeiro trifólio e duas aplicações: uma no surgimento do primeiro trifólio e a outra aplicação no pré-florescimento), com quatro repetições. Em geral, a produção de matéria seca, a atividade da fosfatase ácida, os teores e a eficiência de utilização de fósforo não foram afetados pelos tratamentos de aplicação foliar sob suprimento adequado de P. Sob baixa disponibilidade desse nutriente, a aplicação de fosfito foliar teve efeito negativo sobre estas variáveis. Conclui-se a aplicação foliar de fosfito, comparado ao fosfato, tem efeito depressivo sobre o crescimento do feijoeiro sob baixo suprimento de $\mathrm{P}$. $\mathrm{O}$ fosfito via foliar pouco afeta os teores totais de $\mathrm{P}$, tanto nas raízes quanto na parte aérea, especialmente sob baixo suprimento de $\mathrm{P}$, assim como não proporciona modificações nos teores das frações solúveis de $\mathrm{P}$ nas folhas, em condições de baixo ou adequado suprimento desse nutriente.
\end{abstract}

Palavras-chave: Fosfito de potássio, frações de fósforo, fosfatase ácida, adubação foliar

\begin{abstract}
Currently the use of phosphite as fungicide in agriculture or as a supplementary source of phosphorus (P) has been widespread, however little is known about its effects on phosphate nutrition of important crops such as bean plants. The present work aimed to evaluate the effect of foliar applied phosphite and phosphate on the growth and phosphated nutrition of the bean plant cultivated under low and adequate $\mathrm{P}$ supply conditions. The experiment was conducted in an entirely random layout, in factorial scheme $2 \times 3 \times 2$, where two phosphate concentrations were combined in the nutrient solution $\left(1.5 \mathrm{mg} \mathrm{L}^{-1}=\right.$
\end{abstract}

\footnotetext{
${ }^{1}$ Prof. do Centro de Ciências e Tecnologia Agroalimentar, Universidade Federal de Campina Grande, UFCG, Campus Pombal, Pombal, PB. E-mail: jhosinal_araujo@yahoo.com.br

${ }^{2}$ Prof. do Dept ${ }^{\circ}$ de Ciência do Solo, Universidade Federal de Lavras, UFLA, Lavras, MG. E-mail: vafaquin@dcs.ufla.br

${ }^{3}$ Doutor em Ciência do Solo, Dept ${ }^{\circ}$ de Ciência do Solo, UFLA, Lavras, MG. E-mail: fabriciowlliamavila@yahoo.com.br

${ }^{4}$ Eng $^{\mathrm{o}}$ Agr $^{\mathrm{o}}$, Dept ${ }^{0}$ de Ciência do Solo, UFLA, Lavras, MG. E-mail: thiagopedroso2004@hotmail.com

* Autor para correspondência
} 
low phosphorous and $20 \mathrm{mg} \mathrm{L}^{-1}=$ adequate phosphorous), three foliar application products: $\mathrm{KH}_{2} \mathrm{PO}_{3}$ (monobasic potassium phosphite), $\mathrm{KH}_{2} \mathrm{PO}_{4}$ (monobasic potassium phosphate) and $\mathrm{KCl}$ (potassium chloride) as control and two treatments referring to the number of applications (one application, at the appearance of the first trifoliolate leaf and two applications: one at the appearance of the first trifoliolate leaf and the other application at the pre-flowering), with four repetitions. Overall the dry matter, fosfatase activity, phosphorus content and phosphorus use efficiency was not were affected by the foliar application treatments. Under low readiness of that nutritient the application of foliar phosphite had a negative effect on these variables. It was concluded that foliar applied phosphite has a depressive effect on the growth of the bean plant under low supply of P. The foliar phosphite slightly affects the total concentration of $\mathrm{P}$ in the roots and shoots, especially under low $\mathrm{P}$ supply, and provides no changes in levels of soluble fractions of $\mathrm{P}$ in leaves under conditions of low or adequate supply this nutrient.

Key words: Potassium phosphite, phosphorus fractions, acid phosphatase, foliar fertilization

\section{Introdução}

Os ânions fosfitos $\left(\mathrm{H}_{2} \mathrm{PO}_{3}^{-}, \mathrm{HPO}_{3}^{-2}\right)$ são formas reduzidas de fósforo $(\mathrm{P})$, análogas aos fosfatos $\left(\mathrm{H}_{2} \mathrm{PO}_{4}^{-}, \mathrm{HPO}_{4}^{-2}\right.$ e $\left.\mathrm{PO}_{4}^{-3}\right)$, originadas a partir da redução destes últimos por processos industriais (McDONALD; GRANT; PLAXTON, 2001; THAO; YAMAKAWA, 2009). Atualmente há no mercado diversas formulações à base de fosfito, cuja utilização tem se intensificado nos últimos anos (THAO; YAMAKAWA; SHIBATA, 2009). Estes produtos são comercializados como sais de metais alcalinos obtidos a partir do ácido fosforoso $\left(\mathrm{H}_{3} \mathrm{PO}_{3}\right)$, sendo que os mais produzidos são fosfito de potássio, fosfito de cálcio, fosfito de sódio e fosfito de amônio. Estas formulações são recomendadas como fungicidas ou como suplemento de P, indicadas para diversas culturas (McDONALD; GRANT; PLAXTON, 2001; SCHRÖETTER et al., 2006; MOOR et al., 2009). No Brasil, os fosfitos são comercializados principalmente como soluções de fosfito de potássio, os quais são registrados no MAPA (Ministério da Agricultura, Pecuária e Abastecimento) como fertilizantes para aplicação foliar ou via solo.

Os trabalhos que avaliaram os efeitos dos fosfitos como fonte de $\mathrm{P}$, incluíram principalmente algumas frutíferas e hortaliças como tomate (BERTSCH; RAMÍREZ; HENRÍQUEZ, 2009), alface (THAO; YAMAKAWA; SHIBATA, 2009), morango (MOOR et al., 2009), citrus (ZAMBROSI; MATTOS JUNIOR; SYVERTSEN, 2011), entre outras. As respostas aos fosfitos, observadas nestes trabalhos foram bastante variáveis, sendo que em alguns deles, os efeitos sobre o crescimento e, ou, produção, foram negativos ou nulos (TICCONI; DELATORRE; ABEL, 2001; SCHRÖETTER et al., 2006; THAO et al., 2008; THAO; YAMAKAWA; SHIBATA, 2009; MOOR et al., 2009). Em outros trabalhos, foram relatados incrementos em crescimento ou em produção ou mesmo na qualidade dos produtos agrícolas, pela aplicação de fosfito via solo ou foliar, como em laranja e abacate (ALBRIGO, 1999) e em outras culturas (ÁVILA et al., 2011), especialmente quando estas se encontravam adequadamente supridas com fosfato.

As respostas contraditórias observadas nestes estudos, provavelmente, devem-se às diferenças na sensibilidade entre as espécies a estes íons, assim como às concentrações empregadas e à nutrição fosfatada das culturas testadas (THAO et al., 2008; MOOR et al., 2009). Pouco se sabe como os fosfitos podem atuar negativamente ou positivamente no crescimento e, ou produção das culturas, assim como não há evidências que os fosfitos possam substituir o fosfato no metabolismo vegetal (VARADARAJAN et al., 2002). Ao contrário, em alguns trabalhos observaram-se inibição da atividade de fosfatases ácidas e diminuição das frações solúveis de fósforo nos tecidos vegetais (TICCONI; DELATORRE; ABEL, 2001; LEE; TSAI, 2005).

Conforme Thao e Yamakawa (2009), em geral, os trabalhos com resposta positiva foram realizados 
sob condições de campo, onde as culturas estavam infectadas por Omycetos, principalmente Phytophthora, que são efetivamente controlados por fosfitos (DANIEL; GUEST, 2006), tendo, portanto, o fosfito, nesse caso, atuado de forma indireta. Os efeitos positivos dos fosfitos sobre as culturas poderiam ser em razão, também, da oxidação de fosfito a fosfato por algumas bactérias presentes no ar e, ou, no solo (LOVATT; MIKKELSEN, 2006), principalmente nos ensaios de longa duração, em que o fosfito, depois de oxidado, poderia contribuir com a nutrição fosfatada da cultura.

Em função da escassez de trabalhos dessa natureza com a cultura do feijoeiro e da análise dos resultados de pesquisas obtidos com outras culturas, a hipótese deste trabalho é que o $\mathrm{P}$ na forma de fosfito quando aplicado via foliar, sob deficiência de fósforo, não reverte esta carência para níveis adequados no feijoeiro.

Portanto, no presente trabalho objetivou-se avaliar o efeito do fosfito comparado ao fosfato, aplicados via foliar, sobre o crescimento e a nutrição fosfatada do feijoeiro em condição de baixo e adequado suprimento de $\mathrm{P}$ em solução nutritiva.

\section{Material e Métodos}

O experimento foi conduzido em solução nutritiva, em casa de vegetação do Departamento de Ciência do Solo da Universidade Federal de Lavras, no período de janeiro a março de 2008, com feijoeiro (Phaseolus vulgaris L. cv. BRS Radiante). As sementes de feijoeiro foram germinadas em substrato vermiculita, em bandejas de isopor. Após três dias da emergência, as plântulas foram selecionadas conforme o tamanho e a área foliar e transferidas para vasos de $3 \mathrm{~L}$ contendo solução nutritiva a $50 \%$ de sua força iônica original, com os respectivos tratamentos, recebendo arejamento constante.

A solução nutritiva empregada foi a proposta por Ruiz, Herkehoff Filho e Bunicenha (1988) que,

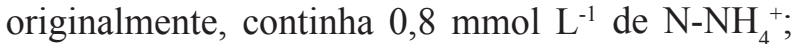
5,4 $\mathrm{mmol} \mathrm{L}^{-1}$ de $\mathrm{N}^{-\mathrm{NO}_{3}}{ }_{3}^{-} ; 0,8 \mathrm{mmol} \mathrm{L}^{-1}$ de $\mathrm{P} ; 2,0$ mmol L-1 de K; 1,7 mmol L-1 de Ca; 0,4 $\mathrm{mmol} \mathrm{L}^{-1}$ de $\mathrm{Mg} ; 0,4 \mathrm{mmol} \mathrm{L}^{-1}$ de $\mathrm{S} ; 40 \mu \mathrm{mol} \mathrm{L} \mathrm{L}^{-1}$ de Fe-EDTA; $19 \mu \mathrm{mol} \mathrm{L}-1$ de B; $7 \mu \mathrm{mol} \mathrm{L}^{-1}$ de Mn; $2 \mu \mathrm{mol} \mathrm{L}^{-1}$ de $\mathrm{Zn} ; 0,5 \mu \mathrm{mol} \mathrm{L}{ }^{-1}$ de $\mathrm{Cu}$ e $0,6 \mu \mathrm{mol} \mathrm{L}{ }^{-1}$ de $\mathrm{Mo}$, com as devidas modificações para o estabelecimento das concentrações de P. Após cinco dias de cultivo, a força iônica da solução foi aumentada para $100 \%$, sendo esta mantida até o final do experimento. O volume de solução dos vasos foi completado diariamente com água deionizada, sendo sua substituição realizada a cada cinco dias e seu $\mathrm{pH}$ corrigido diariamente para valores entre 5,5 e 6,0, pela adição de $\mathrm{NaOH}$ ou $\mathrm{HCl}, 1 \mathrm{~mol} \mathrm{~L}^{-1}$.

O experimento foi conduzido em delineamento inteiramente casualizado, com quatro repetições, em esquema fatorial $2 \times 3 \times 2$, em que foram combinadas duas concentrações de fosfato na solução nutritiva (1,5 $\mathrm{mg} \mathrm{L}^{-1}=$ baixo fósforo e $20 \mathrm{mg} \mathrm{L}^{-1}=$ fósforo adequado), três produtos de aplicação foliar: $\mathrm{KH}_{2} \mathrm{PO}_{3}$ (fosfito monobásico de potássio), $\mathrm{KH}_{2} \mathrm{PO}_{4}$ (fosfato monobásico de potássio) e $\mathrm{KCl}$ (cloreto de potássio) como testemunha e dois tratamentos referentes ao número de aplicações foliares dos produtos (aplicação única no surgimento do primeiro trifólio e duas aplicações, uma no surgimento do primeiro trifólio e a outra aplicação no pré-florescimento). Cada repetição foi constituída por um vaso com duas plantas. As soluções de P (fosfito e fosfato) e a de $\mathrm{KCl}$ foram aplicadas nas concentrações de 40 $\mathrm{mmol} \mathrm{L}^{-1}$ (fontes p.a.), em volume médio de $60 \mathrm{~mL}$ planta $^{-1}$, utilizando-se pulverizador costal manual. A concentração de $\mathrm{P}$ empregada equivale à dose aproximada de $3 \mathrm{~L}$ de fosfito de potássio comercial (131 $\mathrm{g} \mathrm{L}^{-1}$ de $\mathrm{P}$ na forma de fosfito) para $400 \mathrm{~L}$ de calda, normalmente recomendada para a cultura do feijoeiro. $\mathrm{O} \mathrm{KH}_{2} \mathrm{PO}_{3}$ empregado foi obtido pela reação do ácido fosforoso com o hidróxido de potássio. Objetivando otimizar o efeito das aplicações foliares, em cada solução foi adicionado o espalhante adesivo ADESIL $^{\circledR}$ (Agripec), na concentração de $0,2 \mathrm{~mL} \mathrm{~L}^{-1}$ de solução. 
$\mathrm{Na}$ época do florescimento do feijoeiro (10 dias após a segunda aplicação dos produtos de aplicação foliar e 32 dias após o transplantio), foi coletada uma planta de cada repetição, da qual foi retirado o último trifólio fisiologicamente maduro, para a realização do fracionamento do fósforo na matéria fresca destes trifólios e avaliação da atividade das enzimas fosfatase ácida (AFA) nestes tecidos. O fracionamento do $\mathrm{P}$ foi realizado conforme Hogue, Wilcox e Cantlife (1970). Para tanto, foram tomados $0,5 \mathrm{~g}$ de tecido para a avaliação das seguintes frações de $\mathrm{P}$ solúveis em ácido: Pi (fósforo inorgânico), Po (fósforo orgânico) e Pts (fósforo total). A atividade in vivo da fosfatase ácida foi determinada de acordo com os procedimentos descritos em Silva e Basso (1993).

Na planta restante nos vasos de cada repetição foi avaliada a área foliar (AF) utilizando-se o medidor de área portátil Li-Cor modelo Li 3000A, USA e, em seguida, as plantas foram separadas em parte aérea e raízes, e secas em estufa $\left(65^{\circ}-70^{\circ}\right)$ para a avaliação da matéria seca da parte aérea (MSPA) e matéria seca de raízes (MSR). Posteriormente, o material foi moído separadamente, para a determinação de $\mathrm{P}$ nesses tecidos pela digestão nítrico-perclórica (MALAVOLTA, VITTI; OLIVEIRA, 1997). De posse dos dados de MSPA, MSR e dos teores de P total do extrato nítrico-perclórico, foram calculados o acúmulo e a eficiência de utilização de P (EUP), de acordo com Siddiqi e Glass (1981), pela fórmula: $\mathrm{EUP}=(\text { matéria seca total })^{2} /($ acúmulo total de $\mathrm{P})$.

A análise estatística consistiu na análise de variância, teste de médias (Tukey, a 5\%) com auxílio do software SISVAR 4.0. (FERREIRA, 2000) e estudo de correlação entre variáveis com o auxílio do software SAEG 9.1.(SAEG, 2007).

\section{Resultados e Discussão}

A área foliar (AF), a matéria seca da parte aérea (MSPA), a matéria seca de raízes (MSR) e a relação raiz:parte aérea foram influenciados $(\mathrm{p}<0,05)$ pelas concentrações de $\mathrm{P}$ na solução nutritiva $\mathrm{e}$ pelos produtos de aplicação foliar, bem como pela interação entre estes fatores. Para estas variáveis não houve interação significativa do fator número de aplicações dos produtos com os demais fatores (Tabela 1). A área foliar (Figura 1a) e a MSPA (Figura 1b) foram consideravelmente reduzidas pelo baixo suprimento de $\mathrm{P}$ na solução de cultivo. Para a MSR, que também apresentou menor valor com baixo suprimento de $\mathrm{P}$, esse efeito foi menos pronunciado (Figura 1c). A relação raiz:parte aérea (Figura 1d) mostrou comportamento oposto ao observado para AF e MSPA.

A diminuição da área foliar sob baixo suprimento de P é consequência da redução do número de folhas e também da menor expansão foliar (EPSTEIN; BLOOM, 2006). O aumento da relação raiz:parte aérea sob baixa disponibilidade de $\mathrm{P}$ constitui um mecanismo de superação de deficiência de P. Sob tal situação, as plantas destinam parte de fotoassimilados para uma maior produção de raízes, buscando uma maior exploração do seu meio de cultivo e maior absorção de P (MACHADO; FURLANI, 2004).

Comparando-se os tratamentos de aplicação de produtos via foliar, verificou-se que, sob suprimento adequado de $\mathrm{P}\left(20 \mathrm{mg} \mathrm{L}^{-1}\right)$, as variáveis citadas não apresentaram variação significativa entre os tratamentos (Figura 1a-d), concordando com os resultados obtidos por Thao et al. (2008). Sob baixa disponibilidade de $\mathrm{P}$, a área foliar (Figura 1a) não foi afetada pelo fosfito (Phi) foliar, mas foi favorecida pela aplicação foliar de fosfato (Pi). Por outro lado, a aplicação foliar de Phi proporcionou redução significativa na produção de MSPA (Figura 1b), MSR (Figura 1c) e na relação raiz:parte aérea (Figura 1d) em relação ao controle (aplicação de $\mathrm{KCl}$ foliar). Assim, a aplicação foliar de fosfito em plantas deficientes em fósforo pode agravar ainda mais o problema. Resultados semelhantes aos apresentados foram observados em outros trabalhos (SCHRÖETTER et al., 2006; THAO et al., 2008; THAO; YAMAKAWA, 2009; MOOR et al., 2009). 
Tabela 1. Quadrados médios da análise de variância, relacionados à matéria seca da parte aérea (MSPA), matéria seca de raízes (MSR), área foliar (AF) e relação raiz:parte aérea (R/PA), teores de $\mathrm{P}$ na matéria seca da parte aérea (P-MSPA) e nas raízes (P-MSR) e ao acúmulo de P na matéria na MSPA (AcP-MSPA) e nas raízes (AcP-MSR).

\begin{tabular}{lccccc}
\hline \multirow{2}{*}{ Fonte de Variação } & \multicolumn{5}{c}{ Quadrado médio } \\
\cline { 2 - 6 } & G.L & MSPA & MSR & AF & R/PA \\
\hline Concentração de P (C) & 1 & $7.766,83^{* *}$ & $15,25^{* *}$ & $534.734 .252,08^{* *}$ & $0,445^{* *}$ \\
Tratamento foliar (F) & 2 & $31,42^{*}$ & $9,22^{* *}$ & $896.899,39^{*}$ & $0,006^{* *}$ \\
Número de aplicações (N) & 1 & $83,90^{* *}$ & $3,59^{*}$ & $116.821,33^{\text {ns }}$ & $0,000^{\text {ns }}$ \\
C x F & 2 & $13,38^{\text {ns }}$ & $1,40^{\text {ns }}$ & $21.041,02^{\text {ns }}$ & $0,004^{* *}$ \\
C x N & 1 & $17,44^{\text {ns }}$ & $0,14^{\text {ns }}$ & $1.043 .710,08^{\text {ns }}$ & $0,001^{\text {ns }}$ \\
F x N & 2 & $9,07^{\text {ns }}$ & $0,55^{\text {ns }}$ & $1.228 .027,52^{\text {ns }}$ & $0,000^{\text {ns }}$ \\
F x C x N & 2 & $17,67 n^{\text {s }}$ & $1,54^{\text {ns }}$ & $562.141,39^{\text {ns }}$ & $0,001^{\text {ns }}$ \\
Resíduo & 36 & 9,28 & 0,53 & $1.096 .037,20$ & 0,000 \\
CV (\%) & & 11,27 & 13,09 & 18,08 & 9,88 \\
\hline & & P-MSPA & P-MSR & $A c P-M S P A$ & $A c P-M S R$ \\
\hline Concentração de P(C) & 1 & $252,47^{* *}$ & $349,64^{* *}$ & $607.604,63^{* *}$ & $13.978,35^{* *}$ \\
Tratamento foliar (F) & 2 & $0,97^{\text {ns }}$ & $2,08^{\text {ns }}$ & $353,21^{\text {ns }}$ & $12,88^{\text {ns }}$ \\
Número de aplicações (N) & 1 & $0,68^{\text {ns }}$ & $3,31^{\text {ns }}$ & $623,84^{\text {ns }}$ & $18,56^{\text {ns }}$ \\
C x F & 2 & $0,83^{\text {ns }}$ & $1,15^{\text {ns }}$ & $116,03^{\text {ns }}$ & $12,67^{\text {ns }}$ \\
C x N & 1 & $0,12^{\text {ns }}$ & $3,27^{\text {ns }}$ & $3259,14^{\text {ns }}$ & $53,82^{\text {ns }}$ \\
F x N & 2 & $0,79^{\text {ns }}$ & $1,59^{\text {ns }}$ & $2.176,27^{*}$ & $60,74^{\text {ns }}$ \\
F x C x N & 2 & $3,52^{* *}$ & $0,98^{\text {ns }}$ & $2.589,10^{*}$ & $20,52^{\text {ns }}$ \\
Resíduo & 36 & 0,53 & 1,63 & $633,11 \mathrm{~ns}$ & 59,44 \\
CV (\%) & - & 18,00 & 30,74 & 18,41 & 31,66 \\
\hline
\end{tabular}

** , * e ns: significativo, a 1\%, $5 \%$ e não significativo, pelo teste de $\mathrm{F}$, respectivamente.

Fonte: Elaboração dos autores.

De acordo com esses autores acima citados, os fosfitos têm ação repressora dos mecanismos de superação da deficiência de $\mathrm{P}$, como o aumento da atividade da fosfatase ácida, aumento da síntese de transportadores de Pi de alta afinidade e aumento do comprimento, elongação e densidade de pêlos absorventes das raízes, embora não se conheçam os mecanismos exatos pelos quais isso ocorre. Além disso, o fosfito, após absorvido, se mantém estável nos compartimentos celulares, não sendo metabolizado pelas plantas, podendo trazer sérias implicações ao metabolismo vegetal (SINGH et al., 2003; LEE; TSAI, 2005; SCHRÖETTER et al., 2006). Observou-se no presente trabalho que as plantas de feijoeiro após três dias da aplicação foliar com Phi, sob baixo suprimento de P, apresentaram fitotoxidez por fosfito. Os sintomas se manifestaram inicialmente pela presença de clorose generalizada nas folhas jovens. Araújo (2008) observou estes mesmos sintomas em plantas de feijoeiro da mesma cultivar, quando supridas com Phi via solução nutritiva como fonte exclusiva de $\mathrm{P}$, ou quando o Phi foi fornecido sob baixas concentrações de P no meio de crescimento. 
Figura 1. Área foliar (AF)(a), matéria seca da parte aérea (MSPA)(b), matéria seca de raízes (MSR)(c) e relação raíz:parte aérea (d) do feijoeiro em função de tratamentos de aplicação foliar com cloreto de potássio (KCl), fosfito de potássio (Phi) e fosfato de potássio $(\mathrm{Pi})$ e concentrações de fosfato na solução nutritiva, baixa $\left(1,5 \mathrm{mg} \mathrm{L}^{-1}\right)$ e adequada (20 mg L-1). Médias seguidas de mesma letra, minúscula, comparando as concentrações de P na solução para cada produto e, maiúscula, comparando os produtos em cada nível de P, não diferem entre si (Tukey, 5\%).

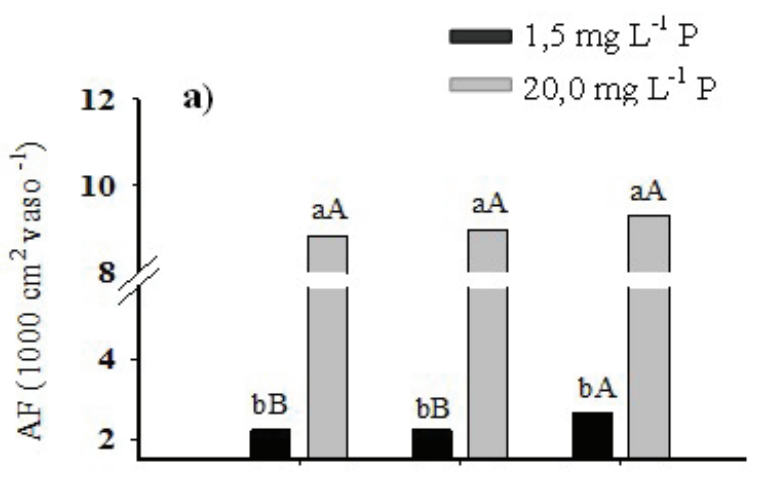

c)

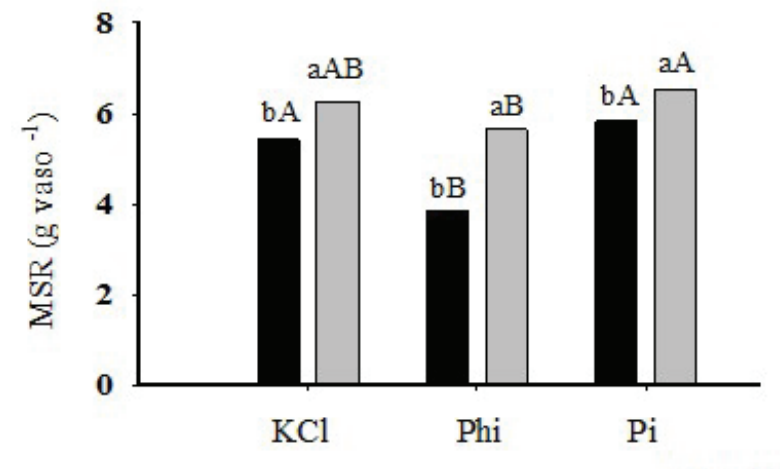

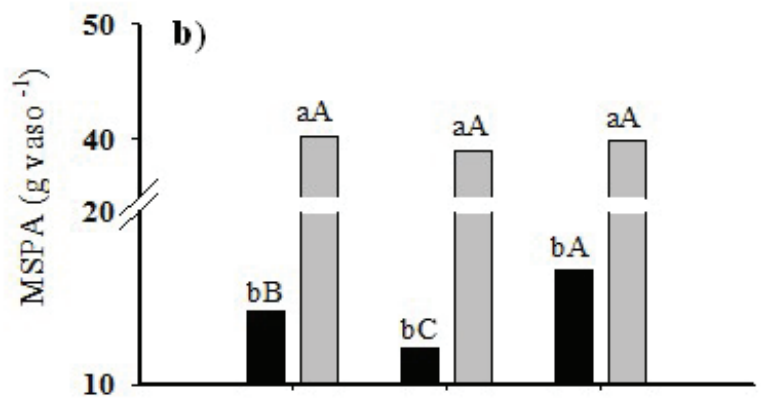

d)

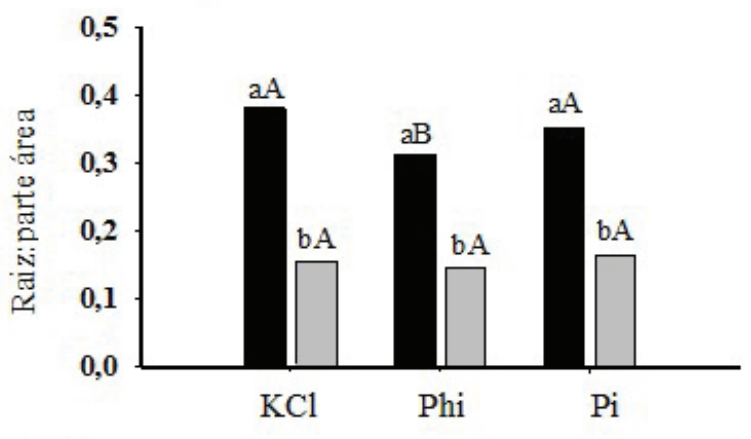

Tratamento foliar

Fonte: Elaboração dos autores.

Os teores de P total (obtido na digestão nítricoperclórica) na MSPA foram influenciados pelas concentrações de $\mathrm{P}$ na solução nutritiva e pela interação tripla entre os três fatores estudados (Tabela 1). O P acumulado na MSPA foi afetado pelas concentrações de $\mathrm{P}$ na solução, pela interação entre as concentrações e o número de aplicações dos produtos de aplicação foliar e pela interação tripla entre os fatores estudados. Os teores de P na MSPA foram, naturalmente, menores sob baixa disponibilidade de $\mathrm{P}$ na solução nutritiva (Figura $2 \mathrm{a}$ e $2 \mathrm{~b}$ ), refletindo em menor acúmulo de $\mathrm{P}$ nestes tecidos sob tal condição (Figura $2 \mathrm{c}$ e $2 \mathrm{~d}$ ).

Quanto aos tratamentos de aplicação de produtos via foliar, verificou-se que, sob baixo suprimento de $\mathrm{P}$ e com duas aplicações foliares, os teores de $\mathrm{P}$ na MSPA foram superiores com o uso de Phi (Figura 2a), o que, provavelmente, reflete o "efeito de concentração", uma vez que o fosfito proporcionou menor produção de MSPA (Figura 1b). 
Figura 2. Teores $(\mathrm{a}, \mathrm{b})$ e acúmulo $(\mathrm{c}, \mathrm{d})$ de fósforo $(\mathrm{P})$ em plantas de feijoeiro, em função de tratamentos de aplicações foliares com cloreto de potássio $(\mathrm{KCl})$, fosfito de potássio (Phi) e fosfato de potássio (Pi) e concentrações de fosfato na solução nutritiva, baixa $\left(1,5 \mathrm{mg} \mathrm{L}^{-1}\right)$ e adequada $\left(20 \mathrm{mg} \mathrm{L}^{-1}\right)$. Médias seguidas de mesma letra, minúscula, comparando o número de aplicações para cada produto e maiúscula, comparando os produtos em cada número de aplicações, não diferem entre si (Tukey, 5\%). Médias seguidas por símbolos diferentes $(+\mathrm{e} *)$, comparando as concentrações de P na solução dentro de cada número de aplicações, diferem entre si (Tukey, 5\%).

$$
1,5 \mathrm{mg} \mathrm{L}^{-1} \mathrm{P}
$$

a)

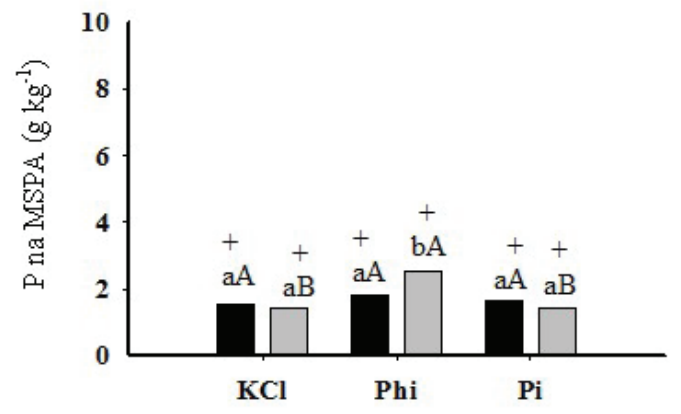

c)

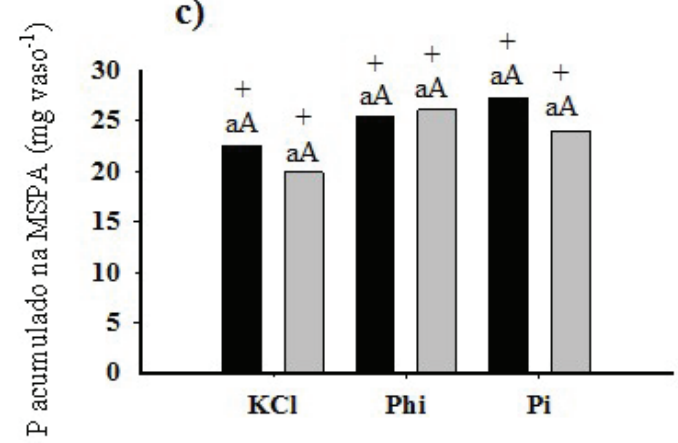

$20,0 \mathrm{mg} \mathrm{L}^{-1} \mathrm{P}$

b)
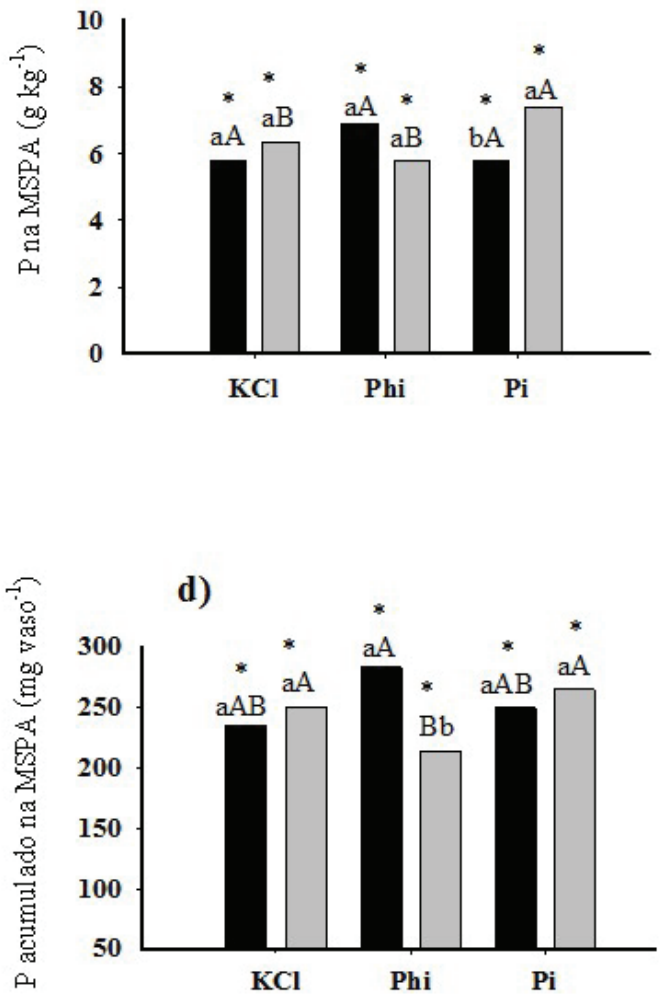

\section{Tratamento foliar}

Fonte: Elaboração dos autores.

Por outro lado, o P acumulado nesses tecidos não sofreu variação significativa nos tratamentos com uma ou duas aplicações foliares (Figura 2c). Sob adequado suprimento de $\mathrm{P}$, o fornecimento de Pi foliar com duas aplicações proporcionou teores mais elevados de $\mathrm{P}$ nos tecidos da parte aérea (Figura 2b). Sob suprimento adequado de $\mathrm{P}$, o fornecimento foliar de Phi em duas aplicações diminui o P acumulado nestes tecidos (Figura 2d), fato esse associado com o efeito detrimental da aplicação foliar de Phi sobre a produção de MSPA
(Figura 1b). Nas raízes, os teores e o acúmulo de $\mathrm{P}$ foram influenciados apenas pela concentração de $\mathrm{P}$ na solução nutritiva, sendo maiores no suprimento adequado de $\mathrm{P}$.

A eficiência de utilização de P (EUP) foi influenciada significativamente pelas concentrações de P na solução nutritiva, pelos produtos de aplicação foliar, pelo número de aplicações dos produtos e pela interação tripla entre estes fatores. Com exceção do tratamento com duas aplicações foliares de Phi sob baixa disponibilidade de $\mathrm{P}\left(1,5 \mathrm{mg} \mathrm{L}^{-1}\right)$, os valores 
de EUP em todos os tratamentos de aplicação foliar, independentemente do número de aplicações, foram sempre superiores sob baixa disponibilidade de P na solução nutritiva (Figura 3a e 3b). Esse resultado indica que, sob alto suprimento de $\mathrm{P}$, grande parte do $\mathrm{P}$ absorvido ficou armazenada nos compartimentos celulares, constituindo o "consumo de luxo" (MALAVOLTA; VITTI; OLIVEIRA, 1997).

Figura 3. Eficiência de utilização de fósforo (EUP) de plantas de feijoeiro, em função de tratamentos de aplicações foliares com cloreto de potássio $(\mathrm{KCl})$, fosfito de potássio (Phi) e fosfato de potássio (Pi) e concentrações de fosfato na solução nutritiva, baixa $\left(1,5 \mathrm{mg} \mathrm{L}^{-1}\right)$ e adequada $\left(20 \mathrm{mg} \mathrm{L}^{-1}\right)$. Médias seguidas de letra minúscula comparam o número de aplicações para cada produto e maiúscula comparam os produtos. Médias seguidas por símbolos diferentes $(+\mathrm{e}$ *), comparando as concentrações de P na solução dentro de cada número de aplicações, diferem entre si (Tukey, 5\%).

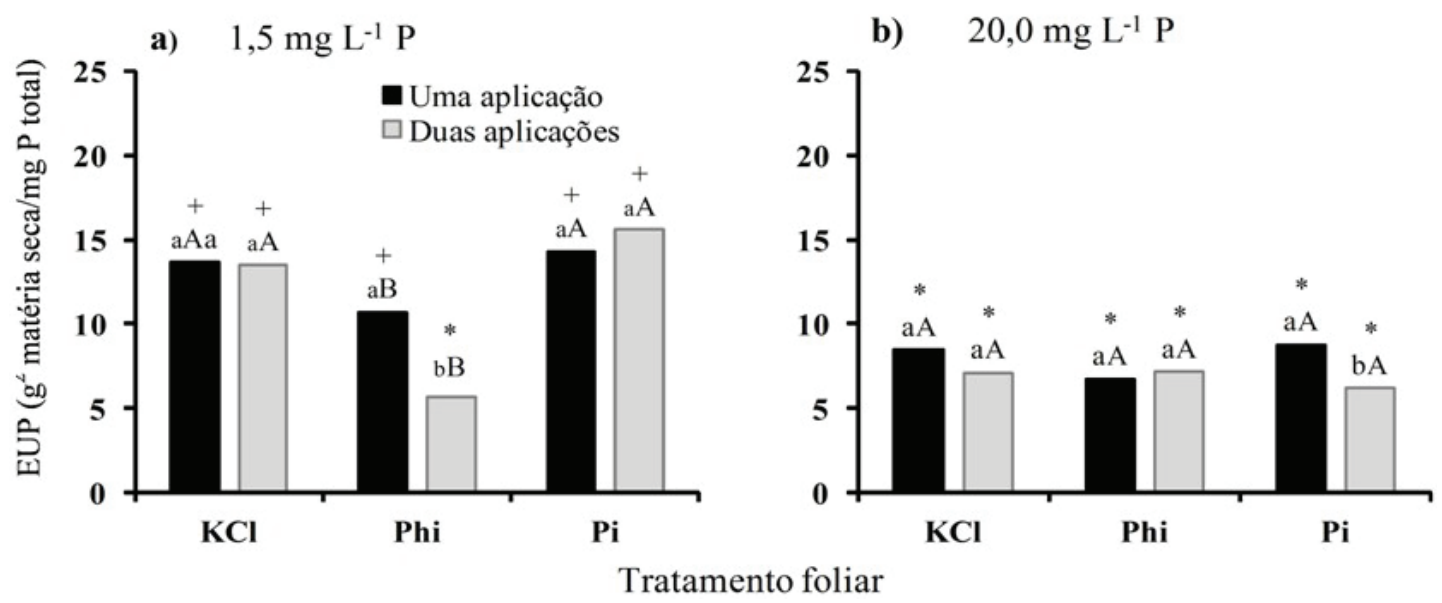

Fonte: Elaboração dos autores.

Com relação aos tratamentos de aplicação foliar, sob baixa disponibilidade de P, a menor EUP foi obtida com a aplicação de Phi, tanto com uma quanto com duas aplicações; com duas aplicações, o efeito foi mais pronunciado (Figura 3a). Sob disponibilidade adequada de $\mathrm{P}$, esse efeito não foi observado, mas o fornecimento de Pi via foliar em duas aplicações reduziu a EUP pelo feijoeiro (Figura 3b), possivelmente devido ao consumo de luxo mencionado.
As concentrações de $\mathrm{P}$ inorgânico $(\mathrm{Pi}), \mathrm{P}$ orgânico e P total (Pts) solúveis em ácido nas folhas diagnósticas do feijoeiro (trifólio recém-maduro, no florescimento) foram afetadas significativamente apenas pelas concentrações de $\mathrm{P}$ na solução nutritiva (Tabela 2). Como já esperado, todas as frações solúveis desse nutriente apresentaram maiores concentrações $(p<0,05)$ sob adequada disponibilidade de P (Figura 4). 
Tabela 2. Quadrados médios da análise de variância, relacionados às frações solúveis de fósforo inorgânico (Pi), fósforo total (Pts) e fósforo orgânico (Po), eficiência de utilização de P (EUP) e atividade da fosfatase ácida (AFA).

\begin{tabular}{lclllll}
\hline \multirow{2}{*}{ Fontes de Variação } & \multirow{2}{*}{ G.L. } & \multicolumn{7}{c}{ Quadrado médio } \\
\cline { 3 - 7 } & & \multicolumn{1}{c}{ EUP } & \multicolumn{1}{c}{ Pi } & \multicolumn{1}{c}{ Po } & AFA \\
\hline Conc. de P (C) & 1 & $282,39^{* *}$ & $1.906 .490,65^{* *}$ & $3.032 .228,09^{* *}$ & $130.191,87^{* *}$ & $124,11^{* *}$ \\
Tratamento foliar (F) & 2 & $61,76^{* *}$ & $116,67^{\text {ns }}$ & $3.069,86^{\text {ns }}$ & $3.845,82^{\text {ns }}$ & $83,67^{* *}$ \\
$\mathrm{~N}^{\text {o }}$ de aplicações (N) & 1 & $18,42^{*}$ & $3.582,47^{\text {ns }}$ & $1.877,70^{\text {ns }}$ & $443,62^{\text {ns }}$ & $3,53^{\text {ns }}$ \\
C x F & 2 & $41,18^{* *}$ & $57,36^{\text {ns }}$ & $4.198,42^{\text {ns }}$ & $4.596,86^{\text {ns }}$ & $87,23^{* *}$ \\
C x N & 1 & $0,04^{\text {ns }}$ & $6.282,78^{\text {ns }}$ & $11.023,14^{\text {ns }}$ & $916,11^{\text {ns }}$ & $8,52^{\text {ns }}$ \\
F x N & 2 & $3,37^{\text {ns }}$ & $213,46^{\text {ns }}$ & $2.796,53^{\text {ns }}$ & $1.695,10^{\text {ns }}$ & $21,59^{* *}$ \\
F x C x N & 2 & $22,79^{* *}$ & $8,40^{\text {ns }}$ & $387,62^{\text {ns }}$ & $431,24^{\text {ns }}$ & $12,10^{\text {ns }}$ \\
Resíduo & 36 & 2,57 & $4.194,82$ & $3.969,10^{\text {ns }}$ & $2.328,25$ & 3,81 \\
CV $(\%)$ & & 16,31 & 28,03 & 16,22 & 27,17 & 5,90 \\
\hline
\end{tabular}

**, * e ns: significativo, a 1\%, 5\% e não significativo, pelo teste de $\mathrm{F}$, respectivamente.

Fonte: Elaboração dos autores.

Figura 4. Teores de fósforo inorgânico (Pi), total (Pts) e inorgânico, solúveis em ácido, em folhas de feijoeiro cultivado em solução nutritiva com baixa $\left(1,5 \mathrm{mg} \mathrm{L}^{-1}\right)$ e adequada $\left(20,0 \mathrm{mg} \mathrm{L}^{-1} \mathrm{P}\right)$ disponibilidade de fósforo. Para cada fração de $\mathrm{P}$, letras minúsculas comparam os produtos de aplicação foliar e minúsculas as concentrações de P na solução nutritiva para cada produto (Tukey, $5 \%$ ).

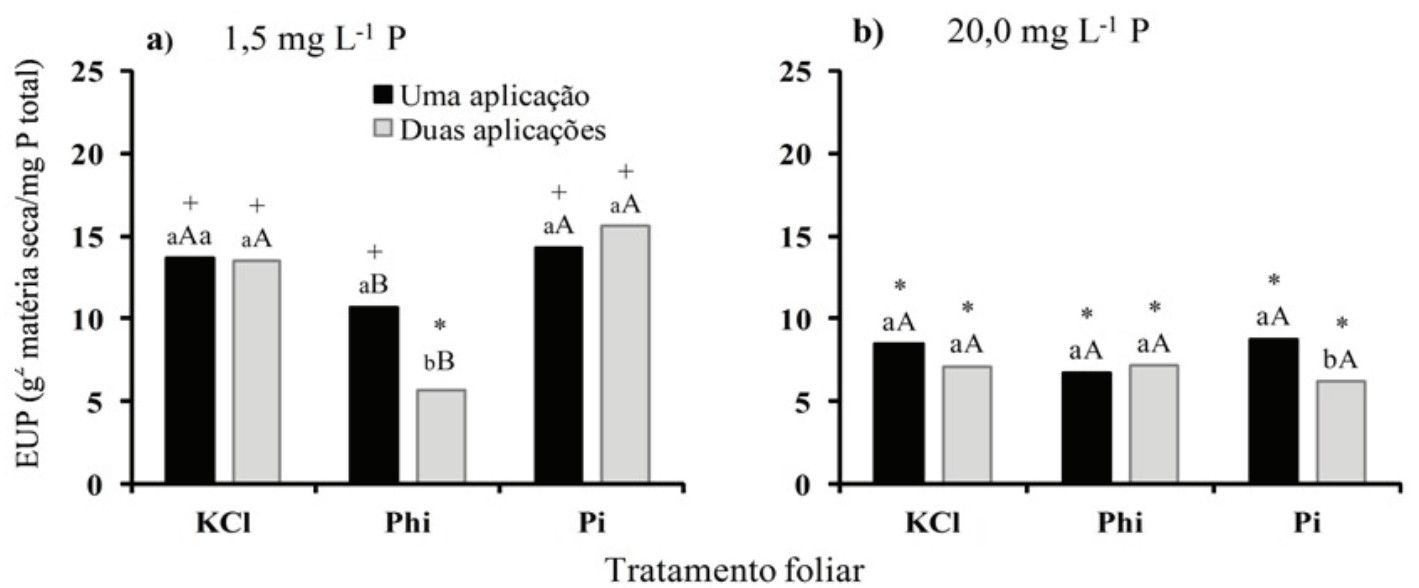

Fonte: Elaboração dos autores.

A atividade da fosfatase ácida (AFA) foi influenciadasignificativamentepelas concentrações de $P$ na solução nutritiva, pelos tratamentos de aplicação foliar, pela interação entre estes fatores e pela interação número de aplicações e tratamentos de aplicação foliar. Sob baixa disponibilidade de P, a AFA foi mais elevada com a aplicação foliar de $\mathrm{KCl}$ (Figura 5a). Por outro lado, em tal condição, a aplicação de Phi foliar reduziu a AFA. Sob suprimento adequado de $\mathrm{P}$ na solução nutritiva, não houve variação significativa na AFA com os tratamentos de aplicação foliar. Comparando-se os tratamentos com baixa e adequada disponibilidade de $\mathrm{P}$ dentro de cada produto de aplicação foliar, verificou-se que, para os tratamentos com $\mathrm{KCl}$ ou Pi foliar, a maior AFA foi obtida com 1,5 mg $\mathrm{L}^{-1}$ de $\mathrm{P}$ na solução nutritiva, enquanto que, com a aplicação foliar de Phi, observou-se efeito 
contrário. Para a interação produtos de aplicação foliar e números de aplicações, independentemente da concentração de $\mathrm{P}$ na solução, a aplicação de Phi reduziu a atividade desta enzima em relação aos tratamentos controle com uma ou duas aplicações e com aplicação de Pi foliar em aplicação única (Figura 4b). A AFA atua na quebra de compostos orgânicos que contêm P para, então, convertê-lo em P inorgânico ou transferir Pi do vacúolo para o citoplasma, principalmente sob baixa disponibilidade de fosfato (SARAPATIKA; DUDOVÁ; KRSKOVÁ, 2004). Entretanto, em condição de baixo suprimento de $\mathrm{P}$, a aplicação de Phi teve efeito inibitório sobre a AFA, fato também observado em outros trabalhos (TICCONI; DELATORRE; ABEL, 2001; SINGH et al., 2003).

Figura 5. Atividade da fosfatase ácida (AFA) em plantas de feijoeiro, em função de tratamentos de aplicações foliares com cloreto de potássio $(\mathrm{KCl})$, fosfito de potássio (Phi) e fosfato de potássio $(\mathrm{Pi})$, concentrações de fosfato na solução nutritiva, baixa $\left(1,5 \mathrm{mg} \mathrm{L}^{-1}\right)$ e adequada $\left(20 \mathrm{mg} \mathrm{L}^{-1}\right)$ (a) e do número de aplicações foliares (b). Médias seguidas de mesma letra minúscula, comparando as concentrações de P na solução ou número de aplicações para cada produto e maiúscula, comparando os produtos em cada concentração de $\mathrm{P}$ ou número de aplicações, não diferem entre si (Tukey, $5 \%$.

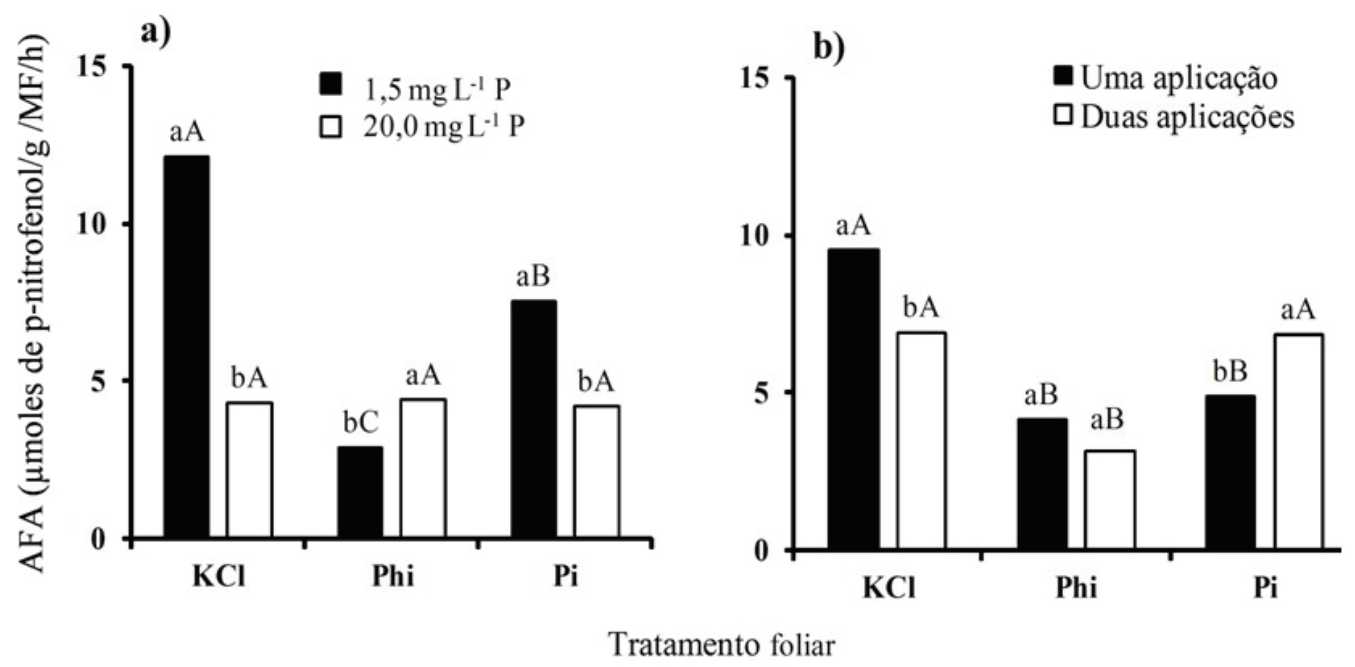

Fonte: Elaboração dos autores.

Os mecanismos fisiológicos e, ou, bioquímicos da toxicidade por fosfitos são pouco compreendidos em vegetais. De acordo com Varadarajan et al. (2002), em fungos, a inibição do crescimento ocorre por causa do acúmulo de poli e pirofosfatos e da inibição de várias enzimas da via glicolítica e também da enzima pentose-fosfato desses organismos. De acordo com Lee e Tsai (2005), embora o P-Phi não seja substrato para enzimas que catalisam as reações de transferência do grupo fosforil, proteínas de plantas e leveduras que se ligam ao $\mathrm{Pi}$, como os transportadores que participam na absorção e na sinalização de respostas moleculares relacionados à deficiência de $\mathrm{P}$, parecem não discriminar entre os ânions Pi e Phi.

O estudo de correlação linear simples entre as variáveis, realizado individualmente para cada número de aplicações dos tratamentos foliares (Tabela 3), confirma, em grande parte, os resultados discutidos anteriormente. A MSPA e a AF correlacionaram-se positivamente com os teores totais de $\mathrm{P}$ (obtido na digestão nítrico-perclórica) e com as frações solúveis de P. Contudo, o Pts e o Pi nas folhas diagnósticas do feijoeiro apresentaram 
relação positiva mais estreita com estas variáveis do que como P total. Em contraposição, AFA e a EUP correlacionaram-se negativamente com as demais variáveis avaliadas embora, na análise com duas aplicações, algumas correlações não tenham sido significativas. Em ambos os números de aplicações, a AFA correlacionou-se positivamente com a EUP, mostrando que a avaliação desta enzima, juntamente com as frações solúveis de $\mathrm{P}$, pode ser útil no diagnóstico nutricional da nutrição fosfatada do feijoeiro quando a forma de P utilizada é o fosfato.

Tabela 3. Coeficientes de correlação linear simples entre produção de matéria seca da parte aérea (MSPA), teor de $P$ na MSPA (P-MSPA), área foliar (AF), fósforo total solúvel em ácido (Pts), fósforo orgânico solúvel em ácido (Po), fósforo inorgânico (Pi), atividade in vivo da fosfatase ácida (AFA) e eficiência de utilização de P (EUP) com uma ou duas aplicações dos tratamentos foliares.

\begin{tabular}{|c|c|c|c|c|c|c|c|}
\hline & MSPA & P-MSPA & $\mathrm{AF}$ & Pts & $\mathrm{Pi}$ & Po & AFA \\
\hline & \multicolumn{7}{|c|}{ Uma aplicação } \\
\hline MSPA & - & - & - & - & - & - & - \\
\hline P- MSPA & $0,91 * *$ & - & - & - & - & - & - \\
\hline $\mathrm{AF}$ & $0,97 * *$ & $0,90^{* *}$ & - & - & - & - & - \\
\hline Pts & $0,97 * *$ & $0,95 * *$ & $0,96 * *$ & - & - & - & - \\
\hline $\mathrm{Pi}$ & $0,97 * *$ & $0,94 * *$ & $0,95^{* *}$ & $0,99 * *$ & - & - & - \\
\hline Po & $0,87 * *$ & $0,87 * *$ & $0,87 * *$ & $0,92 * *$ & $0,86^{* *}$ & - & - \\
\hline AFA & $-0,47^{*}$ & $-0,47^{*}$ & $-0,44^{*}$ & $-0,44^{*}$ & $-0,43^{*}$ & $-0,42 *$ & - \\
\hline \multirow[t]{2}{*}{ EUP } & $-0,70 * *$ & $-0,87^{* *}$ & $-0,73 * *$ & $-0,80 * *$ & $-0,77^{* *}$ & $-0,82 *$ & $0,55^{* *}$ \\
\hline & \multicolumn{7}{|c|}{ Duas aplicações } \\
\hline MSPA & - & - & - & - & - & - & - \\
\hline P- MSPA & $0,83 * *$ & - & - & - & - & - & - \\
\hline $\mathrm{AF}$ & $0,97 * *$ & $0,88^{* *}$ & - & - & - & - & - \\
\hline Pts & $0,90 * *$ & $0,91 * *$ & $0,92 * *$ & - & - & - & - \\
\hline $\mathrm{Pi}$ & $0,88 * *$ & $0,88^{* *}$ & $0,89 * *$ & $0,96 * *$ & - & - & - \\
\hline Po & $0,58 * *$ & $0,64 * *$ & $0,63 * *$ & $0,69 * *$ & $0,47^{*}$ & - & - \\
\hline AFA & $-0,23 \mathrm{~ns}$ & $-0,43^{*}$ & $-0,30 \mathrm{~ns}$ & $-0,37^{*}$ & $-0,43^{*}$ & $-0,05 \mathrm{~ns}$ & - \\
\hline EUP & $-0,40^{*}$ & $-0,73^{* *}$ & $-0,48^{* *}$ & $-0,58$ & $-0,55^{* *}$ & $-0,40^{*}$ & $0,76^{* *}$ \\
\hline
\end{tabular}

**, * e ns: significativo, a $1 \%, 5 \%$ e não significativo, respectivamente.

Fonte: Elaboração dos autores.

Como já discutido anteriormente, o fornecimento de $1,5 \mathrm{mg} \mathrm{L}^{-1}$ de $\mathrm{P}$ na solução nutritiva do feijoeiro foi limitante ao seu crescimento, como uma consequência da deficiência de $\mathrm{P}$ diagnosticado pelos seus baixos teores totais e solúveis e, também, pelo aumento da atividade da fosfatase ácida nos tecidos foliares. Além disso, a alta eficiência de utilização de $\mathrm{P}$ sob baixa disponibilidade de $\mathrm{P}$ (1,5 $\left.\mathrm{mg} \mathrm{L}^{-1}\right)$ indica deficiência severa de $\mathrm{P}$ em relação ao seu adequado suprimento $\left(20 \mathrm{mg} \mathrm{L}^{-1}\right)$. Assim, fica evidente que os efeitos negativos da aplicação foliar de fosfito sob baixo suprimento de fosfato foram devido à deficiência de fósforo na planta.

Os resultados mostram claramente que a aplicação foliar de fosfito sob baixo suprimento de $P$ no meio de cultivo, foi prejudicial ao crescimento do feijoeiro. A atividade da enzima fosfatase ácida (AFA) pode ser uma variável indicadora do efeito negativo do fosfito, entretanto, outras enzimas 
como as envolvidas no metabolismo do fósforo devem ser investigadas. Diante disto, verifica-se a necessidade de um maior aprofundamento dos estudos relacionados com as respostas fisiológicas das plantas tratadas com fosfito.

\section{Conclusões}

Apenas sob baixo suprimento de $\mathrm{P}$, independentemente do número de aplicações, a aplicação foliar de fosfito, tem efeito depressivo sobre o crescimento das plantas de feijoeiro.

O fosfito via foliar pouco afeta os teores totais de $\mathrm{P}$, tanto nas raízes quanto na parte aérea, especialmente sob baixo suprimento de $\mathrm{P}$, assim como não proporciona modificações nos teores das frações solúveis de $\mathrm{P}$ nas folhas, em condições de baixo ou adequado suprimento desse nutriente. A atividade da fosfatase ácida é afetada negativamente pela aplicação foliar de fosfito.

\section{Agradecimento}

À Coordenação de Aperfeiçoamento de Pessoal de Nível Superior (CAPES) pela concessão da bolsa de Doutorado do primeiro autor e ao Departamento de Ciência do Solo pela disponibilização da infraestrutura necessária à execução do trabalho.

\section{Referências}

ALBRIGO, L. G. Effects of foliar applications of urea or nutriphite on flowering and yields of Valencia orange trees. Proceedings of the Florida State Horticultural Society, Tallahassee, v. 112, n. 1, p. 1-4, 1999.

ARAÚJO, J. L. Crescimento e nutrição fosfatada do feijoeiro em função da aplicação via radicular e foliar de fosfito. 2008. Tese (Doutorado em Ciência do Solo) Universidade Federal de Lavras, Lavras.

ÁVILA, F. W.; FAQUIN, V.; ARAUJO, J.A.; MARQUES, D. J.; RIBEIRO JÚNIOR, P. M.; LOBATO, A. K. S.; RAMOS, S. J.; BALIZA, D. P. Phosphite supply affects phosphorus nutrition and biochemical responses in maize plants. Australian Journal of Crop Science, Lismore, v. 5, n. 6, p. 646-653, 2011.
BERTSCH, F.; RAMÍREZ, F.; HENRÍQUEZ, C. Evaluation of phosphite as a root and foliar fertilizer source of phosphorus. Agronomía Costarricense, Costa Rica, v. 33, n. 2, p. 249-265, 2009.

DANIEL, R.; GUEST, D. Defense responses induced by potassium phosphonate in Phytophthora palmivorachallenged Arabidopsis thaliana. Physiology and Molecular Plant Pathology, East Lansing, v. 67, n. 6-5, p. 194-201, 2006.

EPSTEIN, E.; BLOOM, A. Nutrição mineral de plantas: princípios e perspectivas. Londrina: Editora Planta, 2006. $169 \mathrm{p}$.

FERREIRA, D. F. Análises estatísticas por meio do Sisvar para Windows versão 4.0. In: REUNIÃO ANUAL DA REGIÃO BRASILEIRA DA SOCIEDADE INTERNACIONAL DE BIOMETRIA, 45., 2000, São Carlos. Anais... São Carlos: UFSCAR, 2000. p. 255-258.

HOGUE, E.; WILCOX, G. E.; CANTLIFE, D. J. Effect of soil phosphorus levels on phosphate fractions in tomato leaves. Journal American Society for Horticultural Science, Alexandria, v. 95, n. 1, p. 174-176, 1970.

LEE, T. M.; TSAI, P. F. The effects of phosphate on phosphate starvation responses of Ulva lactuca (Ulvales. chlorophyta). Journal of Phycology, Lawrence, v. 41, n. 5, p. 975-982, 2005.

LOVATT, C. J.; MIKKELSEN, R. L. Phosphite fertilizers: what are they? Can you use them? What can they do? Better Crops, Atlanta, v. 90, n. 4, p. 1-11, 2006.

MACHADO, C. T. T; FURLANI, A. M. C. Kinetics of phosphorus uptake and root morphology of local and improved varieties of maize. Scientia Agricola, Piracicaba, v. 6, n. 1, p. 169-76, 2004.

MALAVOLTA, E.; VITTI, G. C.; OLIVEIRA, S. A. Avaliação do estado nutricional das plantas: princípios e aplicações. 2. ed. Piracicaba: Associação Brasileira da Potassa e do Fosfato, 1997. 319 p.

McDONALD, A. E.; GRANT, B. R.; PLAXTON, W. C. Phosphite (Phosphorous acid): Its relevance in the environment and agriculture and influence on plant phosphate starvation response. Journal of Plant Nutrition, New York, v. 24, n. 10, p. 1505-1519, 2001.

MOOR, U.; PÕDMA, P.; TÕNUTARE, T.; KARP, K.; STARAST, M.; VOOL, E. Effect of phosphite fertilization on growth, yield and fruit composition. Scientia Horticulturae, Amsterdam, v. 119, n. 3, p. 264269, 2009.

RUIZ, H. A.; HERKEHOFF FILHO, H. E.; BUNICENHA, J. M. Proporções e concentrações de macronutrientes na formulação de soluções nutritivas 
para culturas de interesse agronômico. V. Feijão. In: REUNIÃO BRASILEIRA DE FERTILIDADE DO SOLO, 18., 1988, Guarapari. Anais... Vitória: SEAG-ES/ EMCAPA/EMATER-ES/SBCS, 1988. p. 1-4.

SARAPATIKA, B.; DUDOVÁ, L.; KRSKOVÁ, M. Effect of $\mathrm{pH}$ and phosphate suply on acid phosphatase activity in cereal roots. Biologia, Bratislava, v. 59, n. 1, p. 127-131, 2004.

SCHRÖETTER, S.; ANGELES-WEDLER, D.; KREUZIG, R.; SCHNUG, E. Effects of phosphite on phosphorus suplly and growth of corn (Zea mays). Landbauforschung Volkenrodxe, Fal Agricultural Research, Bronuschweig, v. 56, n. 3-4, p. 87-99, 2006.

SIDDIQI, M. Y.; GLASS, A. D. M. Utilization index: a modified approach to the estimation and comparison of nutrient utilization efficiency in plants. Journal of Plant Nutrition, New York, v. 4, n. 3, p. 289-302, 1981.

SILVA, F. C.; BASSO, L. C. Avaliação da atividade in vivo da fosfatase ácida da folha na diagnose da nutrição fosfórica em cana-de-açúcar. Revista Brasileira de Ciência do Solo, Viçosa, Campinas, v. 17, n. 3, p. 371375, 1993.

SINGH, V. K.; WOOD, S. M.; KNOWLES, V. L.; PLAXTON, W. C. Phosphite accelerates programmed cell death in phosphate-starved oilseed rape (Brassica napus) suspension cell cultures. Planta, New York, v. 218, n. 2, p. 233-239, 2003.

SISTEMA PARA ANÁLISES ESTATÍSTICAS - SAEG. Versão 9.0. Viçosa. UFV: Fundação Arthur Bernardes, 2007. CD-ROM.
THAO, H. T. B.; YAMAKAWA, T.; SHIBATA, K. Effect of phosphite-phosphate interaction on growth and quality of hydroponic lettuce (Lactuca sativa L.). Journal of Plant Nutrition and Soil Science, Weinheim, v. 172, n. 3, p. 378-384, 2009.

THAO, H. T. B.; YAMAKAWA, T.; SHIBATA, K.; SARR, P. S.; MYINT, A. K. Growth response of komatsuma (Brassica rapa var. peruvirids) to root and foliar applications of phosphite. Plant and Soil Netherlands, v. 308, n. 1, p. 1-10, 2008.

THAO, H. T. B.; YAMAKAWA, T. Phosphite (phosphorous acid): Fungicide, fertilizer or biostimulator? Soil Science and Plant Nutrition, Tokyo, v. 55, n. 2, p. 228-234, 2009.

TICCONI, C. A.; DELATORRE, C. A.; ABEL, S. Attenuation of phosphate starvation responses by phosphite in Arabidopsis. Plant Physiology, Rockville, v. 127, n. 3, p. 963-972, 2001.

VARADARAJAN，D. K.; KARTHIKEYAN，A. S.; MATILDA, P. D.; RAGHOTHAMA, K. G. Phosphite, an analog of phosphate suppresses the coordinated expression of genes under phosphate starvation. Plant Physiology, Rockville, v. 129, n. 3, p. 1-9, 2002.

ZAMBROSI, F. C. B.; MATTOS JUNIOR, D.; SYVERTSEN, J. P. Plant growth, leaf photosynthesis, and nutrient-use efficiency of citrus rootstocks decrease with phosphite supply. Journal of Plant Nutrition and Soil Science, Weinheim, v. 174, n. 3, p. 487-495, 2011. 
